\title{
Double Deeply Virtual Compton Scattering Opportunities at Jefferson Laboratory
}

\author{
Alexandre Camsonne* \\ Jefferson Laboratory Hall A \\ E-mail: camsonne@jlab.org \\ Notice: Authored by Jefferson Science Associates, LLC \\ under U.S. DOE Contract No. DE-AC05-06OR23177 \\ The U.S. Government retains a non-exclusive, paid-up, \\ irrevocable, world-wide license to publish \\ or reproduce this manuscript for U.S. Government purposes.
}

Jefferson Laboratory with the current $12 \mathrm{GeV}$ upgrade and a major program focused on the Generalized Parton Distributions has the opportunity to explore the Doubly Virtual Compton Scattering (DDVCS) process which would allow to probe GPDs surface for skewness di erent from the generalized bjorken variable. Such an experiment requires high luminosity because of a cross section about 100 times smaller than regular Deeply Virtual Compton Scattering. Two letter of intent were submitted to the PAC to carry out this kind of measurement. One in a modified CLAS12 setup in Hall B with a luminosity of $10^{37} \cdot \mathrm{cm}^{2} \cdot \mathrm{s}^{-1}$ and another one using the Solenoidal Large Intensity Device (SoLID) is a solenoidal detector equipped with Gas Elec- tron Multiplier trackers, calorimetry and Cerenkov detectors design to run with luminosity as high as a couple of $10^{38} \cdot \mathrm{cm}^{2} \cdot \mathrm{s}^{-1}$

The 26th International Nuclear Physics Conference

11-16 September, 2016

Adelaide, Australia

${ }^{*}$ Speaker. 


\section{Double Deeply Virtual Scattering}

DDVCS is the most general case of the deeply virtual Compton scattering (DVCS) in which the initial virtual photon transforms into a real photon in the final state. DVCS is the main focus of existing and developing experimental programs since factorization was shown to hold already at electron beam energies of $6 \mathrm{GeV}$ [17]. Several different experimental observables have been investigated, exhibiting expected sensitivity features to specific nucleon GPDs: polarized an unpolarized cross section off the proton [17, ?, 18, 19] and off the neutron [20], beam spin asymmetries off the proton [21, 22], target spin asymmetries off longitudinally [23, 24, 25].

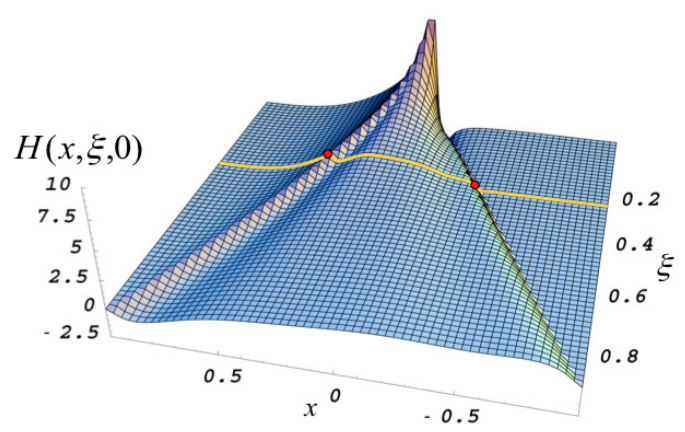

Figure 1: Graphical representation of the DVCS Compton form factor (CFF) showing a typical model for the GPD $H$ at $t=0$; the red points indicates the GPD values involved in the CFF imaginary part, and the yellow line underlines the integral path of the CFF real part.

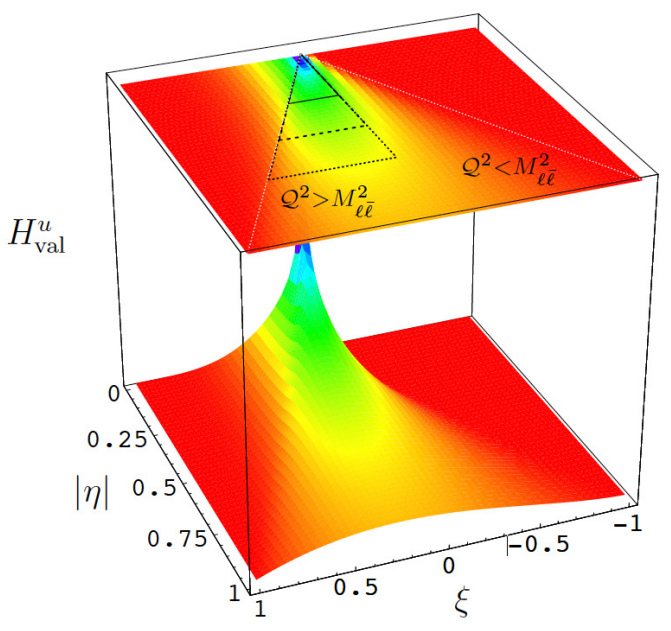

Figure 2: Example of coverage of the GPD surface for different electron beam energies and similar kinematic conditions [16]: $11 \mathrm{GeV}$ (solid line), $25 \mathrm{GeV}$ (dashed line), and $40 \mathrm{GeV}$ (dotted line) in the GPD physics phase space $Q^{2}>Q^{\circ 2}$.

Physics understanding and detection techniques attached to DVCS experiments did reach very high scientific maturity which enables today the ability to take full advantage of the next experimental program generation at JLab $12 \mathrm{GeV}$. Future measurements of the DVCS process will allow for an unprecedented mapping of the nucleon GPDs via the separation of the Compton form factors (CFF), however limited to unambiguous interpretation only along specific correlation lines in the full GPDs kinematic phase-space. For instance, the CFF $\mathscr{H}$ associated with the GPD $H$ and accessible in DVCS polarized cross section or beam spin asymmetry experiments can be written

$$
\mathscr{H}(\xi, t)=\sum_{q} e_{q}^{2}\left\{\mathscr{P} \int_{-1}^{1} d x H^{q}(x, \xi, t)\left[\frac{1}{\xi-x}-\frac{1}{\xi+x}\right]+i \pi\left[H^{q}(\xi, \xi, t)-H^{q}(-\xi, \xi, t)\right]\right\}
$$

where the sum runs over all parton flavors with elementary electrical charge $e_{q}$, and $\mathscr{P}$ indicates the Cauchy principal value of the integral. While the imaginary part of the CFF accesses the GDP values at $x= \pm \xi$, it is clear from Eq. 1.1 that the real part of the CFF is a more complex quantity involving the convolution of parton propagators and the GPD values out-of the diagonals $x= \pm \xi$ (Fig. 1), that is in a domain that cannot be resolved unambiguously with DVCS experiments. Because of the virtuality of the final state photon, DDVCS provides a way to circumvent the DVCS 
limitation $[13,14]$, allowing to vary independently $x$ and $\xi$. Considering the same GPD $H$, the corresponding CFF for the DDVCS process writes

$$
\mathscr{H}(\xi, \eta, t)=\sum_{q} e_{q}^{2}\left\{\mathscr{P} \int_{-1}^{1} d x H^{q}(x, \eta, t)\left[\frac{1}{\xi-x}-\frac{1}{\xi+x}\right]+i \pi\left[H^{q}(\xi, \eta, t)-H^{q}(-\xi, \eta, t)\right]\right\}
$$

involving the additional scaling variable $\eta$ representing here the GPD skewdness. This variable obviously provides the necessary lever arm to investigate the GPD values out-of the diagonals (Fig. 2), that is resolving part of the phase space of interest for the CFF real parts of both DVCS and DDVCS. The kinematically allowed phase space for out-of diagonal exploration is an increasing function of the beam energy but still remains significant at $11 \mathrm{GeV}$ (Fig. 2).

While being theoretically a very attractive process the major experimental difficulties are the reduced cross section induced by the lepton pair decay at the materialization vertex of the final state photon, and the ambiguity between the scattered and decay electrons when investigating the $e^{+} e^{-}$pair production. Additionally, eventual contamination from vector meson decay is putting constraints on the experimental phase space that further reduce the coverage efficiency of an experiment. These latter features did forbid any reliable GPD study from the low statistics data collected with CLAS in a tentative exploratory attempt. This letter-of-intent proposes to solve these issues by taking advantage of the luminosity capabilities of the SoLID spectrometer, and detecting the $\mu^{+} \mu^{-}$di-muon pair from the virtual photon decay.

\subsection{Kinematics}

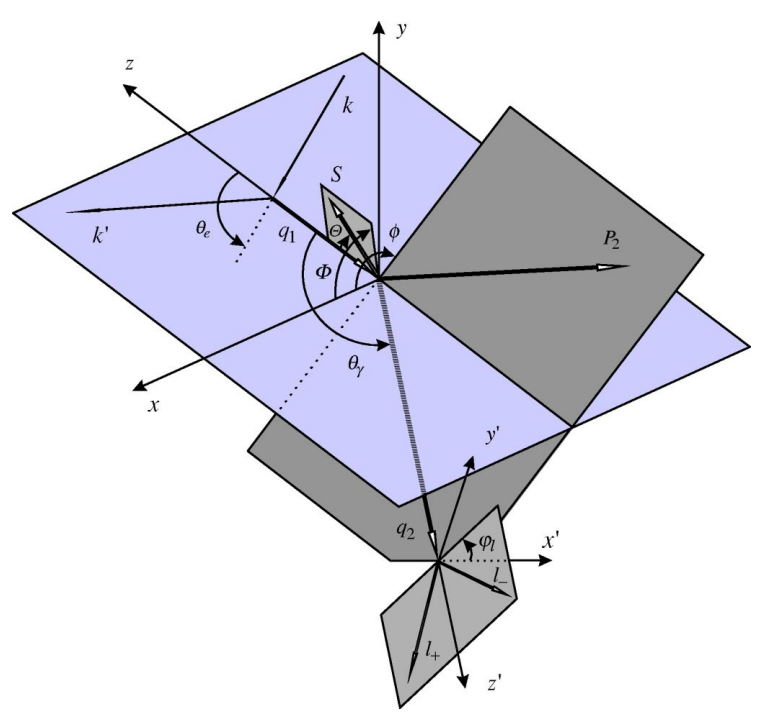

Figure 3: Reference frames for the DDVCS reaction.

The kinematic parametrization of the DDVCS process, expressed in the reference frames of Fig. 3, can be noted

$$
e(k)-e^{\prime}\left(k^{\prime}\right)+p\left(p_{1}\right) \equiv \gamma^{\star}\left(q_{1}\right)+p\left(p_{1}\right) \rightarrow p^{\prime}\left(p_{2}\right)+\gamma^{\star}\left(q_{2}\right) \rightarrow p^{\prime}\left(p_{2}\right)+l^{+}\left(\mu^{+}\right)+l^{-}\left(\mu^{-}\right)
$$

where the photon virtualities write

$$
Q^{2}=-q_{1}^{2}, \quad Q^{\prime 2}=q_{2}^{2} .
$$


Defining the symmetrical variables $p$ and $q$

$$
q=\frac{1}{2}\left(q_{1}+q_{2}\right), \quad p=p_{1}+p_{2},
$$

and the four-momentum transfer to the nucleon $\Delta=p_{1}-p_{2}=q_{2}-q_{1}$ with $t=\Delta^{2}$, the DDVCS scaling variables write

$$
x_{B}=-\frac{1}{2} \frac{q_{1} \cdot q_{1}}{p_{1} \cdot q_{1}}, \quad \xi=-\frac{q \cdot q}{p \cdot q}, \quad \eta=\frac{\Delta \cdot q}{p \cdot q} .
$$

Noting that

$$
q^{2}=-\frac{1}{2}\left(Q^{2}-Q^{2}+\frac{\Delta^{2}}{2}\right)
$$

one gets

$$
\xi=\frac{Q^{2}-Q^{\prime 2}+\left(\Delta^{2} / 2\right)}{2\left(Q^{2} / x_{B}\right)-Q^{2}-Q^{\circ}+\Delta^{2}}, \quad \eta=-\frac{Q^{2}+Q^{\prime 2}}{2\left(Q^{2} / x_{B}\right)-Q^{2}-Q^{\circ}+\Delta^{2}},
$$

which expresses GPDs variables of interest in terms of experimentally measured quantities. The different $Q^{\circ 2}$-dependence in the numerators of $\xi$ and $\eta$ expresses the ability to access out-of diagonals phase space, however limited by experimental and physics constraints.

\subsection{Beam spin asymmetry}

Similarly to the DVCS reaction, the interference amplitude between the BH and DDVCS processes is the observable of interest since it involves linear combinations of Compton form factors, which real an imaginary parts can be accessed in beam charge asymmetry and beam spin asymmetry experiments, respectively, and would ideally be measured by comparing polarized electron and polarized positron scatterings [26]. Considering the harmonic dependence of the cross section, it was shown [16] that the same basic information about GPDs can be obtained from the appropriate moments in $\phi$ or $\varphi_{\mu}$, a feature of particular interest for experimental consistency. Taking advantage of the symmetry properties of the $\mathrm{BH}$ propagators to minimize the $\mathrm{BH}$ contribution, the first $\phi$-moment and $\varphi_{\mu}$-moment of the beam spin asymmetry can be written [16]

$$
\begin{aligned}
\left\{\begin{array}{c}
A_{\mathrm{LU}}^{\sin \phi} \\
A_{\mathrm{LU}}^{\sin \varphi_{\mu}}
\end{array}\right\} & =\frac{1}{\mathscr{N}} \int_{\pi / 4}^{3 \pi / 4} d \theta_{\mu} \int_{0}^{2 \pi} d \varphi_{\mu} \int_{0}^{2 \pi} d \phi\left\{\begin{array}{c}
2 \sin \phi \\
2 \sin \varphi_{\mu}
\end{array}\right\} \frac{d^{7} \vec{\sigma}-d^{7} \overleftarrow{\sigma}}{d x_{B} d y d t d \phi d Q^{\prime 2} d \Omega_{\mu}} \\
& \propto \mathfrak{I m}\left\{F_{1} \mathscr{H}-\frac{t}{4 M_{N}^{2}} F_{2} \mathscr{E}+\xi\left(F_{1}+F_{2}\right) \widetilde{\mathscr{H}}\right\}
\end{aligned}
$$

with the normalization factor given by

$$
\mathscr{N}=\int_{\pi / 4}^{3 \pi / 4} d \theta_{\mu} \int_{0}^{2 \pi} d \varphi_{\mu} \int_{0}^{2 \pi} d \phi \frac{d^{7} \vec{\sigma}+d^{7} \overleftarrow{\sigma}}{d x_{B} d y d t d \phi d Q^{\prime 2} d \Omega_{\mu}}
$$

and where we omit for clarity the $(\xi, \eta, t)$-dependence of the CFF. In the case of a proton target the measurement gives access to the out-of diagonal GPD $H$, while the neutron observable is more sensitive to the $E$ GPD. 
These experimental observables have been obtained using the prescription of Eq. 1.9 for the integration over the angular phase space of the di-muon pair. Similarly to DVCS, the BH process alone on an unpolarized nucleon does not generate beam spin asymmetries. Sizable asymmetries are predicted from the DDVCS and BH interference together with, as expected, a strong sensitivity of the cross section to kinematic conditions.

\section{The Hall B CLAS12 DDVCS setup}

CLAS12 is the upgraded detector for $11 \mathrm{GeV}$ in Hall B. It is a large acceptance detector consisting in a barrel solenoidal detector associated with a more forward toroidal detector based on drift chambers. The particle identification detectors were optimized for the exclusive and semiexclusive reactions program as shown in Fig.4. A modified CLAS12 setup is proposed for DDVCS. It consists in adding a large tungsten plug (Fig. 5) after the calorimeter to shield the drift chambers from all particles while only letting the muons go through giving their momentum analysis in the drift chambers. This setup allows to run at a much higher luminosity than baseline CLAS12 of $10^{37} \mathrm{~cm}^{-2} \dot{s}^{-1}$.

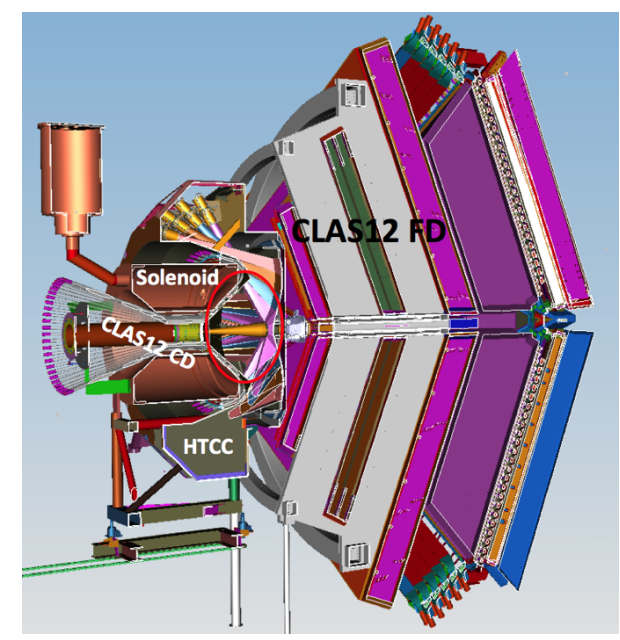

Figure 4: CLAS12 spectrometer

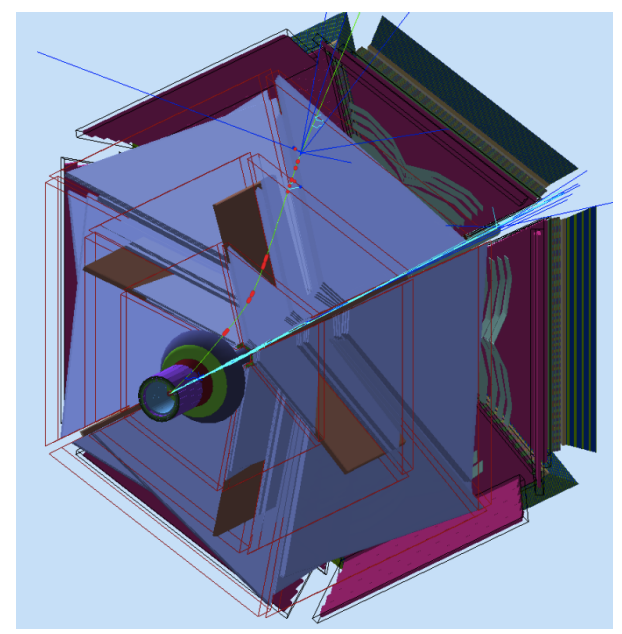

Figure 5: A DDVCS event in the CLAS12 DDVCS setup

The large kinematical reach of the experiment would allow to study the sign change of the DDVCS assymetry for fixed $Q^{2}$ varying $Q^{\prime 2}$ to probe the transition between space-like and timelike DDVCS as shown in Fig. 6 .

\section{The Hall A Solenoidal Large Intensity Device (SoLID) DDVCS setup}

The SoLID spectrometer is based on the CLEO II solenoidal magnet [30] and is already supporting today a unique experimental program [31] [32] in particularly with the $J \Psi$ experiment [35] as shown in Fig.7. The magnet was built in the 1980s by the Oxford Company and installed for CLEO II in 1989 [30]. The main technology developed for the high luminosity purpose of the SoLID detector are Gas Electron Multiplier (GEM) systems arranged in three layers [36]. They 


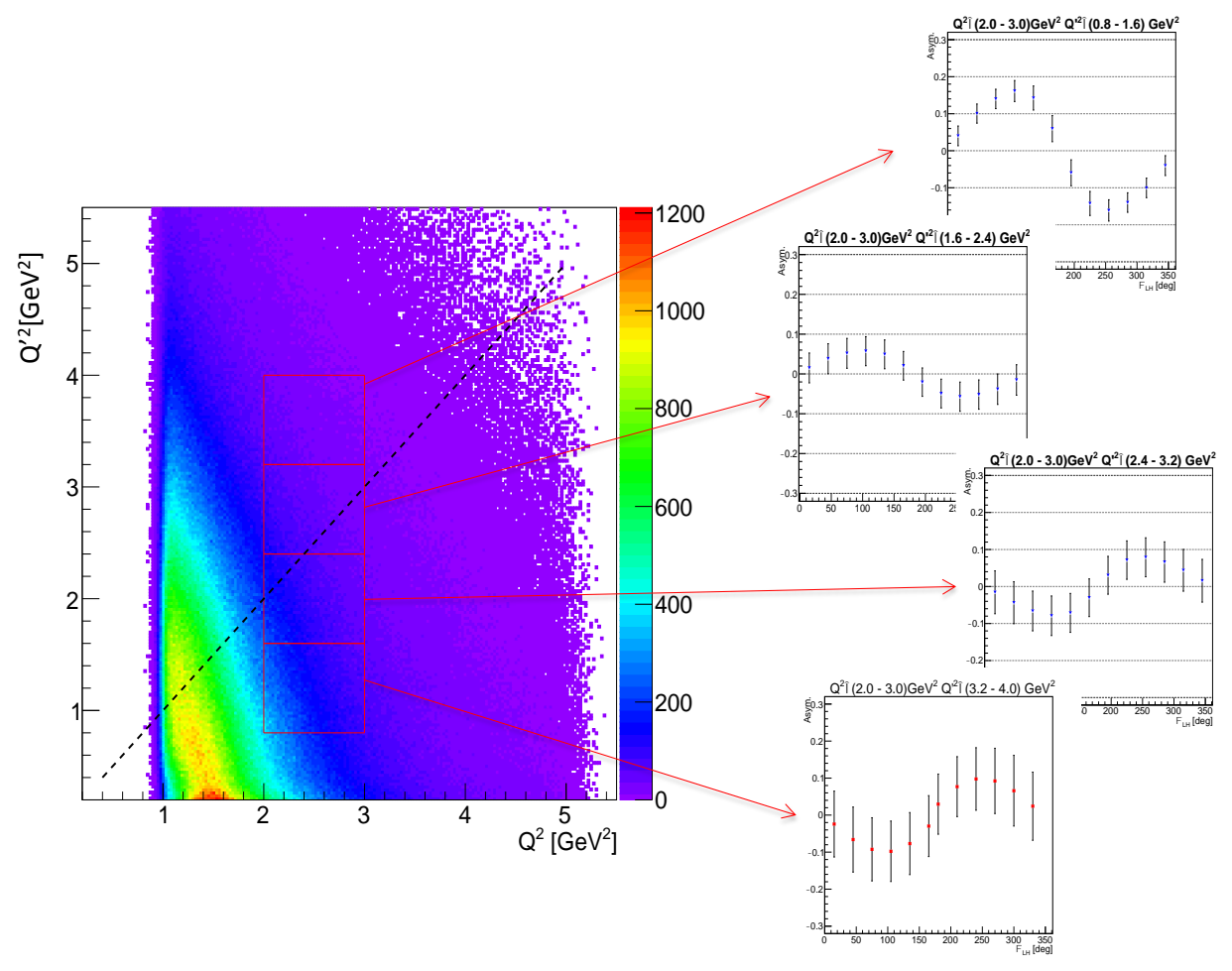

Figure 6: Kinematical coverage of the CLAS12 DDVCS experiment with asymmetries for different bins. One can observe a the sign change of the asymmetry as the transition from space-like to timelike

allow tracking at high rates and are providing the momentum measurement capabilities of the spectrometer. The triple-GEM detectors permit large area detectors with high counting rate capabilities, exceeding $2.5 \mathrm{MHz} / \mathrm{cm}^{2}$ [37], together with excellent spatial resolution $\sim 70 \mu \mathrm{m}$ [38]. The main trigger is based on a shashlyk calorimeter constituting of 1800 preshower and shower hexagonal counters having good radiation hardness properties, moderate energy resolution about $10 \% / \sqrt{E}$, and reasonable intrinsic pion rejection factor $(\sim 10)$. Pion contamination is further reduced by a Light Gas Čerenkov (LGC) detector placed before the calorimeter and constituting of 30 sectors, each read by 9 PMTs for a total number of 270 channels.

We are proposing to in a first step to complement the SoLID detector package for the $J \Psi$ configuration with muon chambers as shown on Fig. 7 and add muon detection capabilities over a large angular phase space. In the case of SoLID, the beam line height constraint allows using the first two layers of iron, leaving part of the iron material and the third layer chambers available. We are thus proposing to recover also the third muon detection layer and provide muon detection at forward angles based on the relocation of the third muon detection layer at the end-cap of SoLID. Such addition would not only establish the capability to achieve a di-muon DDVCS experimental 


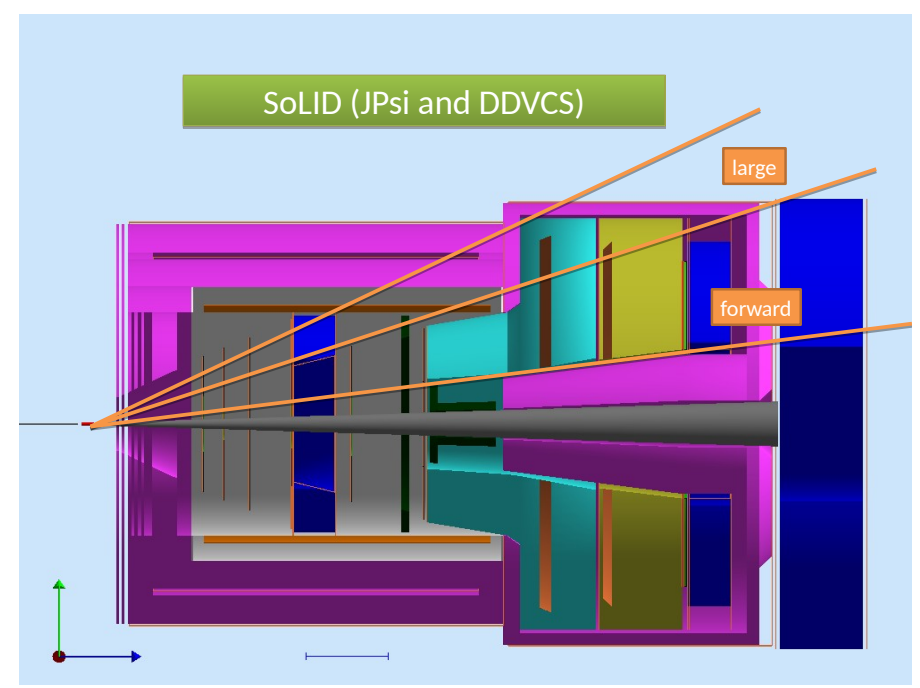

Figure 7: J/ $\Psi$ configuration of the SoLID spectrometer with a muon detector.

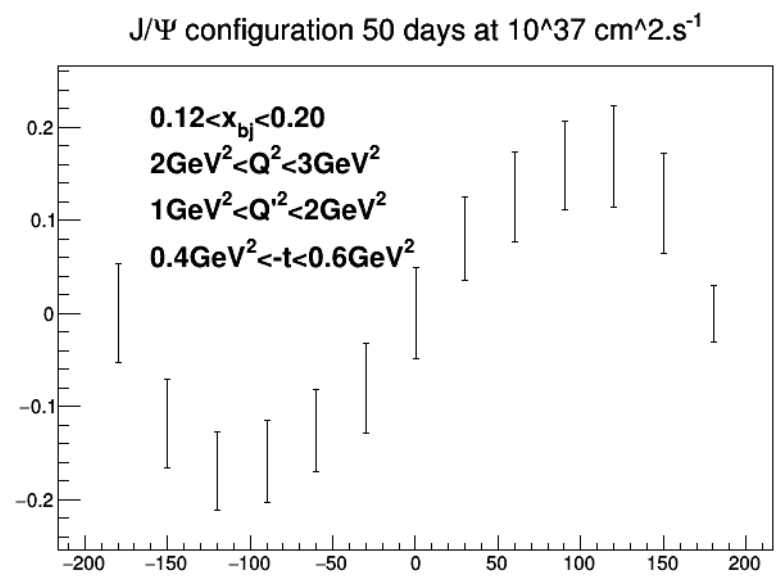

Figure 8: J/ $\Psi$ configuration of the SoLID spectrometer with a muon detector.

program but would also contribute to statistics increase of the $\mathrm{J} / \Psi$ experiment, and would add permanent muon-detection capabilities to SoLID. Expected measured asymmetry is shown in Fig. 8 - A possible dedicated experiment only focusing on muons Fig.9 could allow to run at higher luminosity possibly $10^{38} \mathrm{~cm}^{-2} \dot{s}^{-1}$ increasing the acceptance and kinematical coverage.

\section{Conclusion}

Jefferson Laboratory at $12 \mathrm{GeV}$ along with progress in detector technologies is offering opportunities to carry out the first and unique measurements of DDVCS with the CLAS12 and SoLID apparatuses. This material is based upon work supported by the U.S. Department of Energy, Office of Science, Office of Nuclear Physics under contract DE-AC05-06OR23177. 


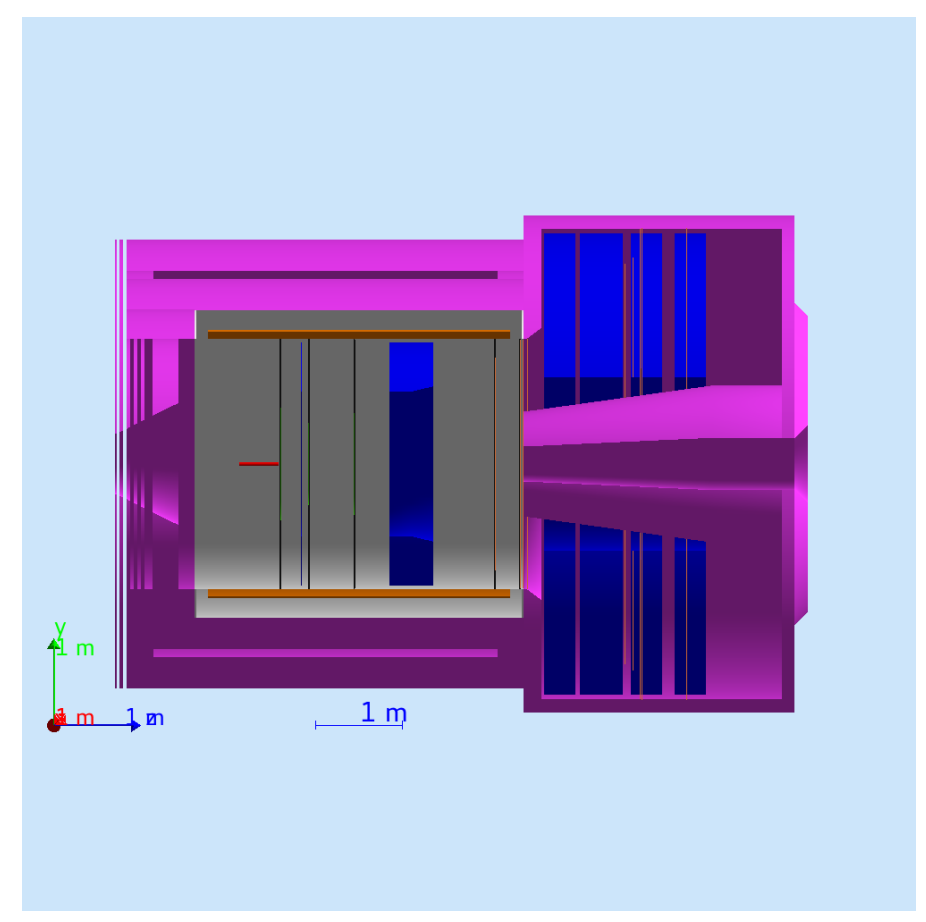

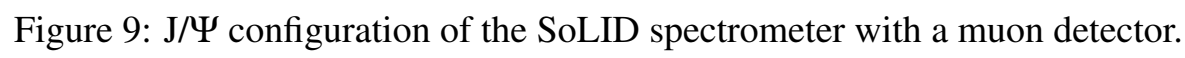

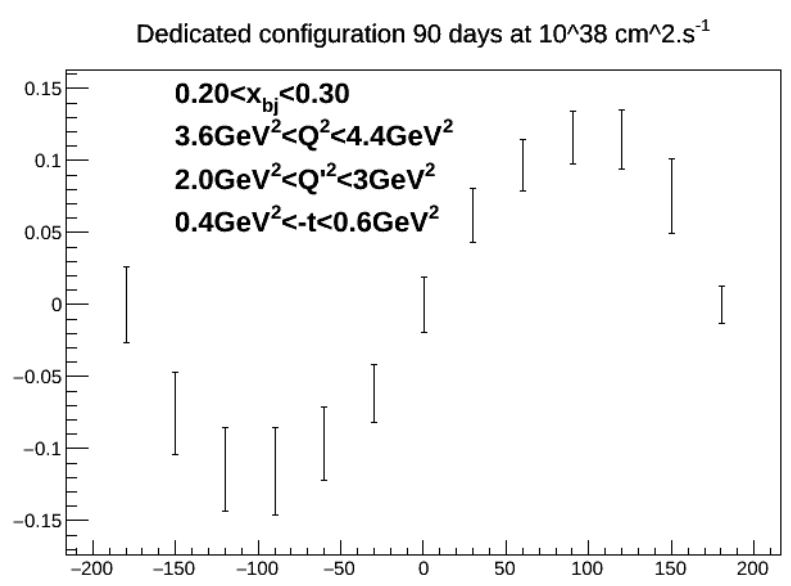

Figure 10: Asymmetry for one bin of dedicated DDVCS experiment with SoLID

\section{References}

[1] D. Müller, D. Robaschick, B. Geyer, F.M. Dittes, J. Hořejši, Fortschr. Phys. 42, 101 (1994).

[2] X. Ji, Phys. Rev. Lett. 78, 610 (1997).

[3] A.V. Radyushkin, Phys. Rev. D 56, 5524 (1997).

[4] M. Diehl, Phys. Rep. 388, 41 (2003).

[5] A. V. Belitsky, A. V. Radyushkin, Phys. Rept. 418, 1 (2005). 
[6] X. -D. Ji, Phys. Rev. D 55, 7114 (1997).

[7] M. Polyakov, Phys. Lett. B 555, 57 (2003).

[8] M. Burkardt, Phys. Rev. D 62, 071503(R) (2000).

[9] J.P. Ralston, B. Pire, Phys. Rev. D 66, 111501(R) (2002).

[10] M. Diehl, Eur. Phys. Jour. C 25, 223 (2002).

[11] A.V. Belitsky, D. Müller, Nucl. Phys. A 711 (2002) 118c.

[12] A.V. Belitsky, D. Müller, A. Kirchner, Nucl. Phys. B 629, 323 (2002).

[13] M. Guidal, M. Vanderhaeghen, Phys. Rev. Lett. 90, 012001 (2003).

[14] A. V. Belitsky, D. Müller, Phys. Rev. Lett. 90, 022001 (2003).

[15] M. Vanderhaeghen, P.A.M. Guichon, M. Guidal, Phys. Rev. D 60, 094017 (1999).

[16] A. V. Belitsky, D. Müller, Phys. Rev. D 68, 116005 (2003).

[17] (Hall A DVCS Collaboration) C. Muñoz Camacho et al., Phys. Rev. Lett. 97, 262002 (2006).

[18] (CLAS Collaboration) H.S. Jo et al., arXiv:hep-ex 1504.02009, (2015).

[19] (Hall A DVCS Collaboration) M. Defurne et al., arXiv:nucl-ex 1504.05453, (2015).

[20] (Hall A DVCS Collaboration) M. Mazouz et al., Phys. Rev. Lett. 99, 242501 (2007).

[21] (CLAS Collaboration) S. Stepanyan et al., Phys. Rev. Lett. 87, 182002 (2001).

[22] (CLAS Collaboration) F.X. Girod et al., Phys. Rev. Lett. 100, 162002 (2008).

[23] (CLAS Collaboration) S. Chen et al., Phys. Rev. Lett. 97, 072002 (2006).

[24] (CLAS Collaboration) E. Seder et al., Phys. Rev. Lett. 114, 032001 (2015).

[25] (CLAS Collaboration) S. Pisano et al., Phys. Rev. D 91, 052014 (2015).

[26] E. Voutier, Nuclear Theory, Edts. A. Georgieva and N. Minkov, Heron Press, 33, 142 (2014).

[27] P. Hoodbhoy, X. Ji, Phys. Rev. D 58, 054006 (1998).

[28] M. Burkardt, Int. J. Mod. Phys. A 18, 173 (2003).

[29] K. Goeke, M.V. Polyakov, M. Vanderhaeghen, Prog. Part. Nucl. Phys. 47, 401 (2001).

[30] Y. Kubota et al., Nucl. Inst. Meth. A 320, 66 (1992).

[31] P. Souder, JLab Proposal E12-10-007 (2010).

[32] J.P. Chen, H. Gao, X. Jiang, J.C. Peng, X. Qian, JLab Proposal E12-10-006 (2010).

[33] J.P. Chen, Y. Qiang, W. Yan, JLab Proposal E12-11-007 (2011).

[34] K. Allada, J.P. Chen, H. Gao, Z.E. Meziani, JLab Proposal E12-11-108 (2011).

[35] K. Hafidi, X. Qian, Z.E. Meziani, N. Sparveris, Z. Zhao, JLab Proposal E12-12-006 (2012).

[36] K. Gnanvo et al., Nucl. Inst. Meth. A 782, 77 (2015).

[37] C. Altunba et al., Nucl. Inst. Meth. A 490, 177 (2002).

[38] B. Ketzer et al., Nucl. Inst. Meth. A 535, 314 (2004). 